\title{
Correlation of Estrogen with Serum Insulin and Blood Glucose Levels in Post-menopausal Women
}

\author{
${ }^{*}$ Yeasmin $N^{1}$, Akther $Q S^{2}$, Hasan $M^{\beta}$, Rabbani $R^{4}$, Rabbani $R^{5}$, Afroz $R^{6}$
}

\begin{abstract}
Hyperglycemia is a major risk factor for cardiovascular diseases in postmenopausal women. Increased incidence of cardiovascular diseases in postmenopausal women may be due to hyperglycemia caused by lower level of estrogen hormone. This cross sectional study was conducted in the Department of Physiology, Dhaka Medical College, Dhaka, Bangladesh during the period of January to December 2011 to observe the correlation of estrogen with fasting serum insulin (FSI) and fasting blood glucose $(F B G)$ levels in postmenopausal women. A total of 90 women were selected from different areas of Dhaka city, among them, 60 postmenopausal women of age group 50 to 60 years were taken as study group and 30 apparently healthy premenopausal women of age group 20 to 30 years were included as comparison group. The study parameters fasting blood glucose level was estimated by enzymatic method in both groups. Serum insulin level was estimated by Enzyme Linked Immunosorbent Assay (ELISA) and serum estrogen level by RIA method in order to assess the hormonal level of both groups. Data was analyzed by Unpaired Student's 't' test and Pearson's correlation co-efficient $(r)$ test as applicable. Mean serum fasting insulin level and mean blood glucose level was higher in postmenopausal women than premenopausal and result was statistically significant. In postmenopausal women serum estrogen level was lower than
\end{abstract}

1. ${ }^{*}$ Dr. Nahid Yeasmin, Assistant professor, Department of Physiology,

Dhaka Medical College, Dhaka. e-mail: masudaatiqua237@gmail.com

2. Dr. Qazi Shamima Akhter, Professor, Head,

Department of Physiology, Dhaka Medical College, Dhaka.

3.Dr. Sayeeda Mahmuda, Associate professor, Department of Pathology, National Institute of Ophthalmology and Hospital, Sher-E- Bangla Nagar, Dhaka.

4. Dr. Mahmudul Hasan, Lecturer, Department of Physiology, Dhaka Medical College, Dhaka.

5.Dr. Rukhshana Rabbani, Assistant Professor, Department of Radiotherapy, Dhaka Medical College.and Hospital.

6. Dr. Rumana Afroz, Assistant Professor, Department of Pharmacology, Dhaka Medical College, Dhaka.

${ }^{*}$ Corresponding Author premenopausal and serum estrogen level showed negative correlation with serum fasting insulin level. Blood glucose level also showed negative correlation with serum estrogen level. All these correlation were statistically non-significant. It may be concluded that the serum fasting insulin and blood glucose levels are significantly higher in postmenopausal women that may be due to low level of estrogen.

Key word: Fasting serum insulin, Fasting blood glucose, Estrogen, Postmenopausal women.

\section{INTRODUCTION:}

Menopause is defined as the permanent cessation of menstruation resulting from the loss of follicular activity. It is recognized by the presence of amenorrhea for 12 consecutive months without any pathological and physiological factors .A new hormonal pattern is established at menopause, which is characterized by high levels of follicle stimulating hormone (FSH), luteinizing hormone (LH) and low level of estrogen.1 Menopause has a wide starting age range, but usually be expected in the range of $42-58$ years.

Following menopause due to lack of estrogen, women have increased risk for insulin resistance or hyperglycemia, central obesity, hyperlipidemia, and hypertension.2 Among these factors the insulin resistance or hyperglycemia is the key factor in the development of type 2 diabetes mellitus, heart diseases and end stages renal diseases. 3

Diabetes mellitus (DM) is one of the common non-communicable diseases and it is one of the most challenging health problems in the twenty first century. 4 There are two general types of diabetes mellitus, one is type 1 or insulin dependent DM (IDDM) and other is type 2 or non-insulin dependent DM. Among them, type 2 diabetes mellitus is more common and about 90 to $95 \%$ of all cases of diabetes mellitus. 5

According to American Diabetes Association, diagnostic criteria of diabetes mellitus are fasting blood glucose $\geq 7.0$ $\mathrm{mmol} / \mathrm{l}$ or 2 hours after $75 \mathrm{gm}$ glucose $\geq 11.1 \mathrm{mmol} / \mathrm{l}$ or random blood glucose $\geq 11.1 \mathrm{mmol} / \mathrm{l}$ or HbAlc $\geq 6.5 \% .6$

According to International Diabetic Federation (IDF), about 24.4 million diabetic people in USA, 65.1 million in India and 5.1 million in Bangladesh. This number is gradually increasing day by day will rise to 592 million by the year 2035 representing about $8.8 \%$ of the world population. By the year 2035, the number of diabetic people will be about 29.7 million in USA, 109.2 million in India and 16.8 million in Bangladesh.7

Insulin is a hormone produced by beta cells a specialized kind of cells in the pancreas. Insulin reduces blood glucose by 
inducing glucose uptake in insulin sensitive tissue such as skeletal muscle, fat cells and heart. In the skeletal muscle insulin prompts glucose uptake by stimulating translocation of the GLUT4 glucose transporter to the plasma membrane. Insulin also inhibits glucose production in liver, kidney and small intestine.8 Insulin resistance occurs when the insulin sensitive tissue loses response to insulin. The basic effect of insulin resistance on glucose metabolism is to prevent the uptake and utilization of glucose by most cells of the body. As a result, blood glucose concentration increases, cell utilization of glucose falls and utilization of fats and proteins increase. Plasma insulin concentration usually increases as a compensatory response by the pancreatic beta cells for diminished sensitivity of target tissues to the metabolic effects of insulin. As a result raising blood glucose and stimulating a compensatory hyperinsulinemia.9- 11

Diabetes mellitus (DM) is common in postmenopausal women and is a major risk factor for cardiovascular diseases, the leading cause of mortality in women. In the United States, at least 1.8 million women of reproductive age (18-44 years) are estimated to have DM compared with 3.8 million among women aged 45-65. 12

Some epidemiological studies observed that postmenopausal women with diabetes have a coronary heart diseases (CAD) related mortality rate 4to 7 times higher than that of non-diabetic postmenopausal women. Again premenopausal women with diabetes have CAD related mortality 2 to 4 times higher than that of non-diabetic premenopausal women. 13

The postmenopausal year is associated with rise of fasting insulin and glucose levels. The marked decline in endogenous estrogen production after menopause, resulting in increased relative androgenicity and change in body composition is suggested to influence pancreatic $\beta$-cell function, insulin induced glucose transport and hepatic glucose output.14

Estrogen is a steroid hormone produced primarily in the ovaries and too much lesser extent in other cells like fat tissue. Estrogen production from the ovaries declines around and after menopause. 15

Estrogen may have beneficial effect on insulin sensitivity via a number of possible mechanisms direct effects on insulin and glucose homeostasis, involvement in adipose tissue metabolism and body composition and pro-inflammatory markers. 16

During menopause decreases the production of estrogen and followed by post-glucose challenge hyperinsulinemia, implying insulin resistance. In postmenopausal women due to lack of estrogen decreased insulin mediated glucose uptake via an impaired insulin stimulated translocation of GLUT4 to the plasma membrane and decreased protein expression of glycogen synthase. 17

Some other studies also reported that serum insulin and blood glucose levels are higher in postmenopausal women than that of premenopausal women.1,15,17 Opposite finding was reported by some investigators they did not find any significant difference in serum insulin level between premenopausal and postmenopausal women.18

Increased incidence of coronary artery diseases (CAD) resulting from increased levels of serum insulin and blood glucose, have been reported. But very few publication about the effect of estrogen on serum insulin and blood glucose levels in postmenopausal are available. Therefore, this study has been designed to observe the correlation of estrogen with serum insulin and blood glucose levels in postmenopausal women.

\section{METHOD:}

This cross sectional study was conducted in the Department of Physiology, Dhaka Medical College, Dhaka, during the period of January 2011 to December 2011. A total number of 90 female subjects were selected from different areas of Dhaka city by simple random sampling. Among them, 60 postmenopausal women with age ranging from 50 to 60 years were taken as study group and 30 apparently healthy premenopausal women with age ranging from 20 to 30 years were included as control group for comparison. Subjects having history of heart, liver, kidney diseases and women taking hormone replacement therapy steroid, alcohol user, and smoker were excluded from the study. After selection of the subjects, the objectives, nature, purpose and benefit of the study were explained to the subjects in details. They were encouraged for voluntary participation. They were also allowed to withdraw from the study whenever they feel like. Ethical permission was taken from ethical committee of Dhaka medical College. Written informed consents were taken from the participants. Detailed medical history, menstrual history and family history of the subjects were taken and recorded in a pre-designed data collection form. Then with all aseptic precautions $5 \mathrm{ml}$ of venous blood from each subject was collected after an overnight fast (at least 12 hours) from median cubital vein by disposable plastic syringe. Blood was allowed to clot and then centrifuged at a rate of $3000 \mathrm{rpm}$ and supernatant clear serum was separated. Serum was taken in to eppendrof tube and was preserved in refrigerator in Department of Physiology of Dhaka Medical College, Dhaka. Then estimation of serum estrogen level was done by Radioimmunoassay (RIA) method in the Department of Nuclear Medicine, Dhaka Medical College. Estimation of serum insulin level was done by Enzyme Linked Immunosorbent Assay (ELISA) method in the Department of laboratory of National Institute of ENT, Dhaka and fasting blood glucose was estimated by glucose oxidase method in the Department Dhaka Medical College in both groups. Statistical analysis was done by Unpaired Student's ' $t$ ' test. Correlation was analyzed by Pearson's correlation co-efficient (r) test. P value $<0.05$ was taken as of significance. 
Table I: Age, fasting serum insulin (FSI) and fasting blood glucose (FBG) level in premenopausal and postmenopausal women

\begin{tabular}{ccccc}
\hline Groups & $\mathrm{n}$ & Age (years) & $\mathrm{FSI}(\mu \mathrm{IU} / \mathrm{ml})$ & $\mathrm{FBG}(\mathrm{mg} / \mathrm{dl})$ \\
\hline $\mathrm{A}$ & 30 & $28.77 \pm 6.66$ & $10.05 \pm 3.07$. & $87.90 \pm 27.45$ \\
$\mathrm{~B}$ & 60 & $53.90 \pm 5.75$ & $19.10 \pm 8.41$ & $118.14 \pm 50.17$ \\
\hline \multicolumn{5}{l}{ Statistical analysis } \\
\hline Groups & $\begin{array}{c}\text { Age } \\
\text { (p value) }\end{array}$ & $\begin{array}{c}\text { FSI } \\
\text { (p value) }\end{array}$ & $\begin{array}{c}\text { FBG } \\
\text { (p value) }\end{array}$ \\
\hline A vs. B & $0.0001^{* * *}$ & $0.0001^{* * *}$ & $0.003^{* *}$
\end{tabular}

Unpaired Student's ' $t$ ' test was performed to compare between groups. The test of significance was calculated and $\mathrm{p}$ values $<0.05$ was accepted as level of significance.

Group A : Premenopausal women $\mathrm{n}=$ Number of subject

Group B : Postmenopausal women $\mathrm{ns}=$ Not significant

${ }^{* * *}=$ Significant at $\mathrm{P}<0.001$

In this study, the mean $( \pm \mathrm{SD})$ of age was $28.77 \pm 6.66$ years in group A and $53.90 \pm 5.75$ years in group $B$. The age in group B was higher than that of group $A$ and the result was statistically significant $(\mathrm{p}<0.0001)$.

Figure-1: Mean fasting serum insulin level in premenopausal and postmenopausal women

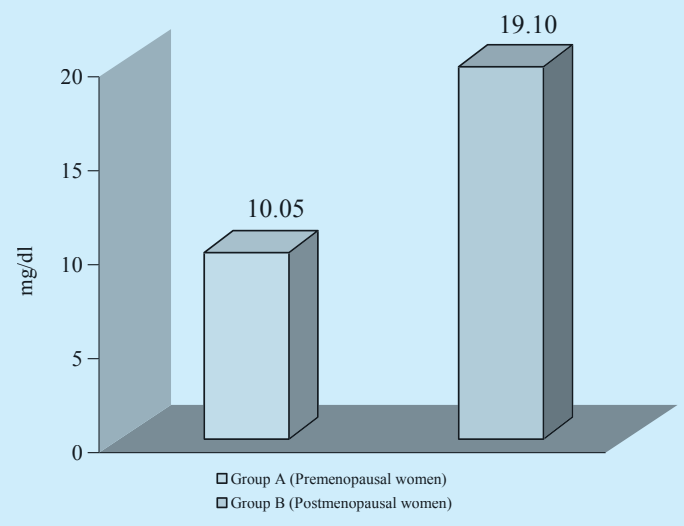

Figure- 1 shows the mean $( \pm S D)$ of serum insulin level $10.05 \pm 3.07 \mu \mathrm{IU}$ in group $\mathrm{A}$ and $19.10 \pm 8.41 \mu \mathrm{IU}$ in group $\mathrm{B}$. The serum insulin level was higher in postmenopausal women (group B) than those of premenopausal women (group A) and the result was statistically significant $(\mathrm{p}<0.001)$.
Figure- 2: Mean serum fasting blood glucose level in premenopausal and postmenopausal women.

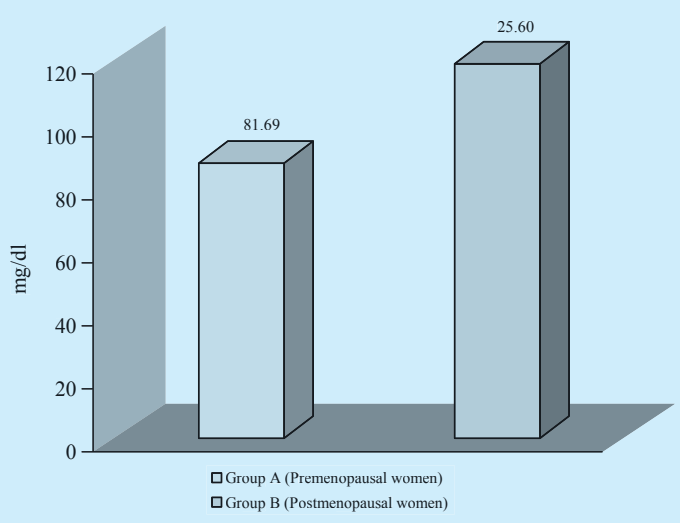

Figure- 2 shows the mean $( \pm$ SD) of blood glucose level $87.90 \pm 27.45$ $\mathrm{mg} / \mathrm{dl}$ in premenopausal women (group A) and 118.14 50.17 $\mathrm{mg} / \mathrm{dl}$ in postmenopausal women (group B). The result was significantly $(\mathrm{p}<0.01)$ higher in postmenopausal women (group B) than those of premenopausal women (group A). (Figure- 2)

Table II: Serum estrogen level in premenopausal and postmenopausal women

\begin{tabular}{ccc}
\hline Groups & $\mathrm{n}$ & $\begin{array}{c}\text { Estrogen } \\
(\mathrm{pg} / \mathrm{ml})\end{array}$ \\
\hline A & 30 & $81.69 \pm 36.61$ \\
B & 60 & $25.60 \pm 17.35$ \\
\hline
\end{tabular}

Serum

Groups estrogen

\begin{tabular}{lc} 
& (p value) \\
\hline $\mathrm{A}$ vs $\mathrm{B}$ & $0.003^{* *}$ \\
\hline
\end{tabular}

Results are expressed as mean $\pm \mathrm{SD}$

Unpaired Student's ' $t$ ' test was performed to compare between groups. The test of significance was calculated and $p$ values $<0.05$ was accepted as level of significance.

Group A : Premenopausal women $n=\quad$ Number of subjects Group B :Postmenopausal women $\quad * *=\quad$ Significant at $\mathrm{P}<0.01$ ${ }^{* * *}=$ Significant at $\mathrm{P}<0.0$

The mean $( \pm S D$ ) of estrogen level was $81.69 \pm 36.61 \mathrm{pg} / \mathrm{ml}$ in group $A$ and $25.60 \pm 17.35 \mathrm{pg} / \mathrm{ml}$ in group B. The estrogen level was lower in group $B$ than that of group A and the result was statistically significant $(\mathrm{p}<0.001) \quad($ Table-II). 
Table-III: Correlation of serum estrogen level with biochemical parameters in postmenopausal women

\begin{tabular}{lcc}
\hline Parameters & \multicolumn{2}{c}{ Group B $(\mathrm{n}=60)$} \\
& $\mathrm{R}$ & $\mathrm{p}$ \\
\hline FSI & 0.062 & $0.0638 \mathrm{~ns}$ \\
FBG & & \\
\hline
\end{tabular}

Table-III shows the pearson's correlation coefficient $(r)$ test to compare relationship between parameters. The test of significance was calculated and $\mathrm{p}$ value $<0.05$ was accepted as level of significance

Group B : Postmenopausal women

$\mathrm{n}=$ Number of subjects

$\mathrm{ns}=$ Not significant

The serum estrogen level negative correlation $(r=-0.062)$ with serum insulin level in postmenopausal women and result was statistically non-significant. In postmenopausal women the blood glucose level showed negative correlation $(r=-0.063)$ with serum estrogen level and result was statistically non-significant.

\section{Figure- 3: Correlation of serum estrogen and fasting serum insulin level in postmenopausal women}

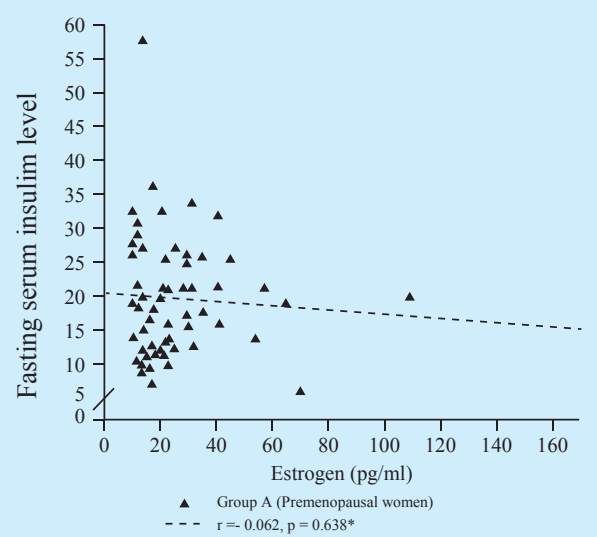

Figure- 3 shows the serum estrogen level negative correlation $(r=-$ 0.062 ) with serum insulin level in postmenopausal women and result was statistically non-significant. In postmenopausal women the blood glucose level showed negative correlation $(r=-0.063)$ with serum estrogen level and result was statistically non-significant.

\section{Figure- 4: Correlation of serum estrogen and fasting blood glucose in postmenopausal woman}

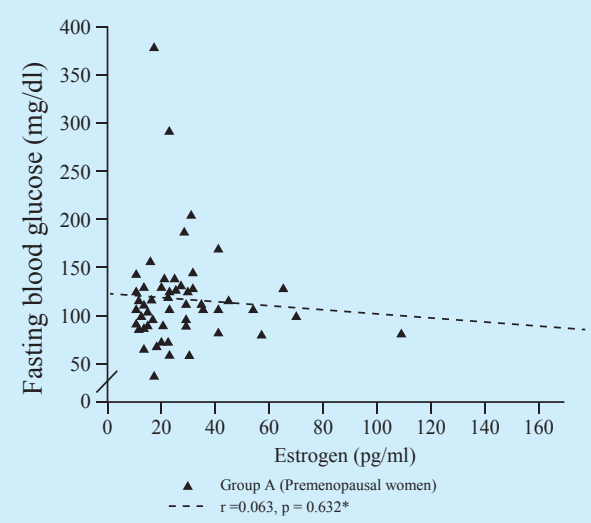

Figure- 4 shows the serum estrogen level negative correlation ( $r=-$ 0.062 ) with serum insulin level in postmenopausal women and result was statistically non-significant. In postmenopausal women the blood glucose level showed negative correlation $(r=-0.063)$ with serum estrogen level and result was statistically non-significant.

\section{DISCUSSION:}

In the present study the levels of fasting serum insulin and blood glucose in healthy premenopausal women were almost within normal range and also similar to reported by the several investigators from abroad.1-4

The mean serum insulin level in postmenopausal women was higher than that of premenopausal women and result was statistically significant. Similar types of findings were reported by different researchers of different countries.8,13,15 On the contrary, similar observations were made by other researchers but they did not find any significant difference in fasting serum insulin level. This result may be due to small sample size in their study.18 Again, in our study, fasting serum insulin level showed negative correlation with serum estrogen level in postmenopausal women. Similar type of result was found by other workers. 13

The level of fasting blood glucose in postmenopausal was higher than those of premenopausal women and result was statistically significant. Similar types of findings were reported by different researchers of different countries13,15 Again, in our study, blood glucose level showed negative correlation with serum estrogen level in postmenopausal women and result was statistically non-significant. .

Many explanations are suggested by different investigators regarding development of insulin resistant in postmenopausal women. It has been suggested that estrogen has regulatory influence on the function of pancreatic $\beta$-cells. Estrogen regulates insulin action directly via action on insulin sensitive tissues or indirectly by regulating factors 
like oxidative stress, which plays important role in development on insulin resistance. In skeletal muscle estrogen is thought to have positive effect on insulin signaling and GLUT4 expression. Estrogen enhances insulin-induced glucose transport by activating phosphatidyl inositol-3 kinase signaling, leading to translocation of GLUT4 to the plasma membrane. This process increase insulin mediated glucose uptake into cells.16,17

Again, increased accumulation of central abdominal fat in women at the time of menopause is associated with a decline in the production of a protein called adiponectin. Adiponectin, which is produce by fat cell is important for glucose and fatty acid metabolism. It makes muscle and liver cells more sensitive to the action of insulin. Moreover it stimulates glucose utilization in muscle, thus decreases blood glucose level. So decreased level of adiponectin in postmenopausal women are associated with increased blood glucose level.18,19

It is well known that estrogen has anti-inflammatory properties. In postmenopausal women due to lacking of estrogen there are increased cytokine levels, including TNF, IL-1 and IL-6. These inflammatory markers are associated with insulin resistance either directly by affecting the insulin signaling pathway or indirectly by stimulation of inflammatory pathway.20-22

In the present study, both fasting serum insulin and blood glucose levels are higher in postmenopausal women than premenopausal women. This is most likely due to lower level of estrogen, as the measured value of estrogen was lower in postmenopausal women than premenopausal women. Furthermore, in the present study, fasting serum insulin and blood glucose levels, showed negative correlation with serum estrogen level in postmenopausal women. These correlations further support these findings. But exact mechanism is not elucidated by this type of study due to time and financial constraints.

From this study, it can be concluded that higher values of fasting serum insulin and blood glucose may present in postmenopausal women may be due to their lower level of estrogen hormone.

\section{REFERENCE:}

1. ZA,Begum,S,Ferdousi,S, Begum, Ali,T, \& Begum,A. FVC,FEV,and FEV/FVC in postmenopausl women and their relationship with serum progesterone and estrogen level..J Bangladesh Soc Physiol.2009; 4(1): 7-13.

2. Hidalgo LA, Chedraul PA, Morocho N,Alvarado M, Chavez D and Hue A. The metabolic syndrome among postmenopausal women in Ecuador. Gynecol Endocrinol .2006; 22(8): 447-54.

3. Heidery R, Masoumeh s, Mohammad T, Kateyour R, Noushin Nizal S. Metabolic syndrome in menopausal transition: Isfahan healthy heart program, a population based study. Diabeto Metab syndr.2010;2:59-67.

4. Sadu MM, Protasiewicz DC, Firanescu AG. Data regarding the prevalence and incidence of diabetes mellitus and prediabetes. Rom J Diabetes Nutr Metab Dis.2016;23(1):95-103.

5. Hall, JE. Guyton \& Hall Text Book of Medical Physiology. 12th ed. India :Elsevier limited;2011; p. 618.

6. American Diabetes Association.Classification and diagnosis of Diabetes.Diabetes Care.2015; 38(1): S8-16.

7. Guariguata .L, Whiting. DR, Hambleton I. Global estimates of diabetes prevalence for 2013 and projections for 2035. Diabetes Res Clin Prect. 2013; 103(2014): 137-49.

8. Mercurio V, Carlomango G, Fazio V \& Fazio S. Insulin resistance is it time for primary prevention? World J Cardiol.2012; 4(1):1-7.

9. Pessin. JH \& Sal.Signatiel AR. Signaling pathways in insulin action: molecular targets of insulin resistance. J Clin Ivest.2000; 106(2):64-69.

10. Brand JS,Vaan der Schouw YTand Onland Moret NC.Age at menopause, reproductive life span and type 2 diabetes risk: Result from the EPIC-Inter Act study. Diabetes Care.2013; 36(1):1012-19.

11. Kim C. Does menopause increase diabetes risk? Strategies for diabetes prevention in midlife women. Women Health.2012; 8:155-67.

12. Sathya Bhana CV, Balaji S, Seethalakshni A. Analysis of the degree of insulin resistance in postmenopausal women by using skin temperature measurements and fasting insulin and fasting glucose levels: A case control study.Journal of clinical and diagnostic research.2012;16(10): 1644-47.

13. Duke Appiah,Stephen J andCarlton A. Hornung. Bilateral Oophorectomy and the risk of incident diabetes in postmenopausal women.Diabetes Care.2014;37(1):725-33.

14. Kumagai S,Holmang A, Bjorntorp.The effects of estrogen and progesterone on insulin sensitivity in female .Acta Physol Scand. 1993;149: 91-97.

15. Surapon Tangvarasiittichi. Oxidative stress, insulin resistance, dyslipidemia and type 2 diabetes mellitus. World journal of Diabetes 2015; 6(3):456-80.

16. Cambell SE,Febbraio MA. Effect of the ovarian hormones on GLUT4 expression and contractionstimulalated glucose uptake. AMJ Physiol Endocrinol Metab.2002; 282:E1139-E1146.

17. Ying Zhang,Barbara V. Howard, Linda D Crawn, Jeunliang Yeh and Carl F Schaefer. The effect of estrogen use on levels of glucose and insulin and risk of type 2 diabetes in American Indian postmenopausal women.Diabetes 
Care.2002; 25(3):500-4.

18. Baily CJ, Ahmed Sorour H. Role of ovarian hormones in the long term control of glucose homeostasis and effect of insulin secretion. Diabetologia.1980; 19:475-78.

19. Mankowska, Lena,N and Grazyna, S.Adiponectin and metabolic syndrome in women at menopause. Diabetic News.2011.

20.Koyong Park,Myron Gross,Duk-Hee Lee,Paul Holvoet, John H Himes, James M Shikany.Oxidative stress and Insulin Resistance. Diabetic Care.2009; 32(1):1302-307.

21. Cari de Luca \& Jerrold M, Olefsky. Inflammation and insulin resistance.NIH public Access.2008; 582(1):97-105.

22. Ana L,Guadarrana Lopex,Roxana Valdes Ranos and Beatrize Martinez Carrillo. Type 2 diadetes,PUFAS and vitamin D: Their relation to inflammation.Journal of Immunology Research.2014;(2014):8-13 